\title{
A Novel, Low-Profile, Vertically-Polarized UWB Antenna for WBAN
}

\author{
Mohsen Koohestani, J.-F. Zürcher, Antonio A. Moreira, and Anja K. Skrivervik
}

\begin{abstract}
This paper proposes a novel, low-profile UWB antenna for wireless body area network (WBAN) applications. The antenna has a polarization perpendicular to the body-free-space interface, which is interesting in order to minimize the coupling into the body. Its structure comprises a modified mono-cone with a top-cross-plate and is coaxially fed through the ground plane. The higher frequency band $\left|S_{11}\right|$ performance is due to the mono-cone while the top-cross-plate is responsible for the lower frequency band. This plate also leads to a height reduction when compared to conventional mono-cone antennas. A comprehensive parametric study is done to provide design guidelines. Both frequency- and time-domain results have been measured and presented to validate the design. Results show that the antenna operates from 3.06 to beyond $12 \mathrm{GHz}$ based on $\left|S_{11}\right| \leq-10 \mathrm{~dB}$, radiates omni-directionally in the $H$-plane, and has a radiation efficiency over $95 \%$. The system-fidelity factor for UWB signals is adequate for pulse transmission. Finally, the influence of the human proximity on the antenna matching was tested. Results show that its impedance is nearly unchanged as compared to free-space.
\end{abstract}

Index Terms-Frequency- and time-domain, low profile, UWB antenna, vertically polarized, wireless body area network.

\section{INTRODUCTION}

$\mathbf{T}$ HE interest for wireless, body-area networks (WBAN) has grown significantly for applications as diverse as healthcare systems, sports monitoring, ambient intelligence, personal entertainment, and military services [1]-[3]. Ultrawideband (UWB) technology has emerged as an attractive solution for WBAN as it offers some benefits such as transmitting signals with an inherent noise-like behavior (due to low EIRP level), reduced probability of detection and intercept, robustness against multipath, and potentially high data rates over short distances.

Manuscript received July 19, 2013; revised October 29, 2013; accepted January 05,2014 . Date of publication January 09,2014 ; date of current version April 03, 2014. This work was supported by the Portuguese Fundação para a Ciência e a Tecnologia (FCT) project PEst-OE/EEI/LA0008/2011, under Grant SFRH/BD/51445/2011.

M. Koohestani is with the Laboratoire d'Electromagnétisme et d'Acoustique, École Polytechnique Fédérale de Lausanne (EPFL), 1015 Lausanne, Switzerland, and also with the Instituto de Telecomunicações, Instituto Superior Técnico, Universidade de Lisboa, 1049-001 Lisbon, Portugal (e-mail: koohestani.mohsen@epfl.ch).

J.-F. Zürcher and A. K. Skrivervik are with the Laboratoire d'Electromagnétisme et d'Acoustique, École Polytechnique Fédérale de Lausanne (EPFL), 1015 Lausanne, Switzerland (e-mail: anja.skrivervik@epfl.ch).

A. A. Moreira is with the Instituto de Telecomunicações, Instituto Superior Técnico, Universidade de Lisboa, 1049-001 Lisbon, Portugal (e-mail: antonio. moreira@1x.it.pt).

Color versions of one or more of the figures in this paper are available online at http://ieeexplore.ieee.org.

Digital Object Identifier 10.1109/TAP.2014.2298886
The antenna plays a critical role for the quality of a WBAN link as the proximity of the body of the wearer may significantly modify characteristics such as their impedance bandwidth, radiation pattern/polarization and efficiency [4]. In the case of UWB antennas, the impact of the wearer on the transient characteristics should also be considered [5]. These antennas are required to have a low backward radiation, low height with compact form, low coupling to the human body, and suitable transient characteristics (high system-fidelity factor). Additional desirable requirement for UWB-WBAN antennas in order to have efficient on-body communication is the polarization [6], which should preferably be perpendicular rather than parallel to the body surface. These features make the design of such antennas quite challenging.

There are several UWB antenna designs available [7]-[11], which meet some of the mentioned requirements. Klemm [7] added a reflector below the antenna feed-line to reduce the backward radiation. The advantage and disadvantage of other ways to achieve directionality such as adding a cavity or shielding plane behind the antenna, or using absorbing materials have also been discussed in that paper. Abbosh [8] corrugated the antenna radiator to make the antenna compact. The antenna studied in [9] was loaded by proper dielectrics to minimize the coupling to the body. See et al. have shown in [10] that body-proximity improves the antenna time behavior; a fact corroborated in [11]. Regarding the antenna polarization, Lim [12] presented a study demonstrating the advantages of using antennas with a polarization perpendicular to the user's surface, for on body communications. This is due to the fact the $E$-fields are normal to the body and tend to propagate along the body surface, while for a parallel polarization the fields couple more to the body, leading to enhanced losses. Moreover, the antenna should be low profile to be easily worn, which is not easy to achieve for the desired polarization.

Some UWB wearable antenna designs are reported in literature [6], [13]-[19] which have polarization perpendicular to the body surface. Chahat [6] proposed a reduced-size printed UWB monopole antenna having an overall height of $10 \mathrm{~mm}$. Kang [13] introduced a novel folded UWB antenna utilizing a bevel edge feeding structure with the overall height of $10 \mathrm{~mm}$. Cara [14] presented a tripod kettle antenna with $10 \mathrm{~mm}$ height covering half of the FCC-defined UWB spectrum. Sanz-Izquierdo [15] reported a UWB button antenna with $12.5 \mathrm{~mm}$ height. Chan [16] proposed a miniaturized modified planar inverted-F antenna with $8.5 \mathrm{~mm}$ height, including the ground and top plate, but due the offset in the feeding structure it has an unsymmetrical radiation pattern. To the authors' best knowledge, the latter design has the smallest height among the recently de- 


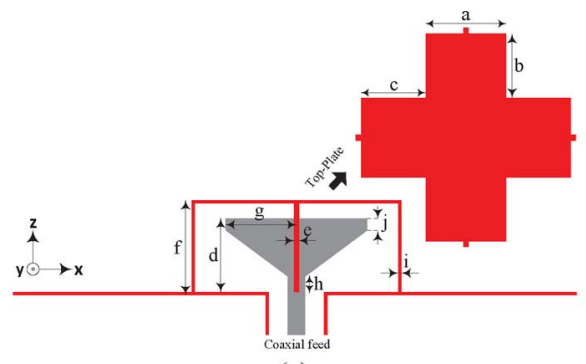

(a)

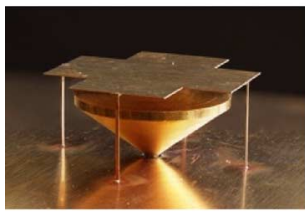

(b)

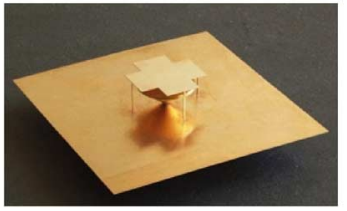

(c)

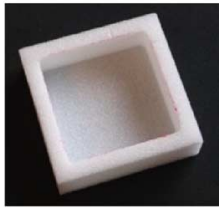

(d)

Fig. 1. Top-cross-plate UWB mono-cone antenna: (a) geometry; (b) close-view; (c) full-view; and (d) designed radome.

TABLE I

PARAMETER VAluES OF THE FABRICATED ANTENNA

\begin{tabular}{c|c|c|c|c|c|c|c|c|c|c} 
Parameter & $\mathrm{a}$ & $\mathrm{b}$ & $\mathrm{c}$ & $\mathrm{d}$ & $\mathrm{e}$ & $\mathrm{f}$ & $\mathrm{g}$ & $\mathrm{h}$ & $\mathrm{i}$ & $\mathrm{j}$ \\
\hline value- $m m$ & 10 & 5 & 5 & 6.8 & 0.25 & 8.5 & 8 & 0.25 & 0.1 & $\mathbf{1}$
\end{tabular}

signed UWB-WBAN vertically polarized antennas. Other reported designs [17]-[19] have larger heights.

In this work, we propose a new low-profile UWB antenna structure that fully meets the above-mentioned WBAN imposed requirements. The physical height of this antenna is small having the same height as in [16] but with symmetrical radiation properties. The characteristics of the proposed antenna are investigated through a parametric study, in order to develop design guidelines. Both frequency- and time-domain results were measured and are presented in terms of $S$-parameters, radiation pattern, radiation efficiency, impulse response and system-fidelity factor. We also investigate the behavior of the antenna in two wearer scenarios, and the effect of the body on the antenna matching was tested.

\section{AnTENNA Structure AND Design}

The idea behind the design of the proposed structure is based on a conventional mono-cone antenna [20]. In order to reduce the height, the mono-cone was modified adding a small cylinder on its top and covering it by a top-cross-plate supported by four legs to further enhance its $-10 \mathrm{~dB}$ impedance bandwidth. The top-cross-plate acts as a capacitive load that produces the lower frequency performance while the higher frequencies behavior is due to the modified mono-cone. The antenna ground and top-cross-plate are made of a thin-layer Beryllium copper with conductivity of $1.5 \times 10^{7} \mathrm{~S} / \mathrm{m}$; the mono-cone is made of Brass with conductivity of $3.8 \times 10^{7} \mathrm{~S} / \mathrm{m}$. It has a low height and a compact form; the dimensions are given in Table I. A photograph of the fabricated antenna is shown in Fig. 1. Since the proposed structure contains some delicate parts, i.e., top-cross-plate legs, a radome was designed to enable employing the antenna in a harsh environment. Therefore, in order to protect the antenna and make it robust, a hollow-cube made of a 5-mm thick foam
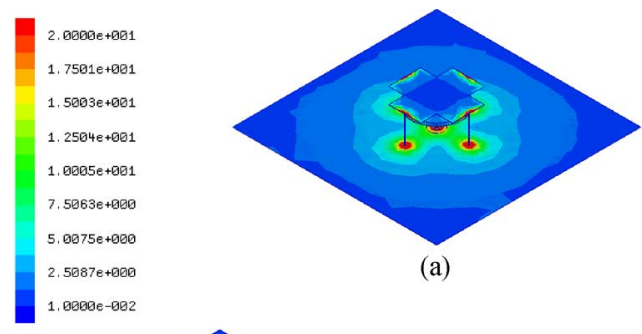

(a)

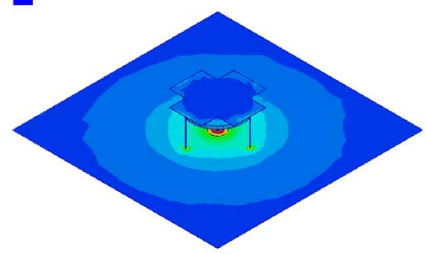

(b)

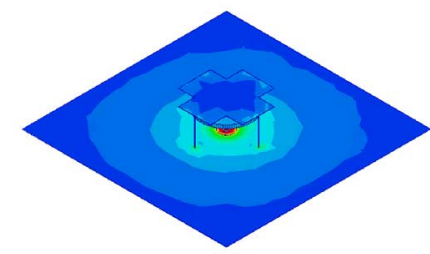

(c)
Fig. 2. Current distribution of the antenna at: (a) $3.1 \mathrm{GHz}$; (b) $6.85 \mathrm{GHz}$; and (c) $10.6 \mathrm{GHz}$.

material (relative permittivity 1.07 and loss-tangent of 0.004 ), as shown in Fig. 1(d), was fabricated. A standard high-strength two-component epoxy (UHU plus) adhesive was used to glue the radome to the proposed structure.

In order to demonstrate the antenna operation, the current distribution on the antenna at $3.1 \mathrm{GHz}$ (lower frequency), $6.85 \mathrm{GHz}$ (middle frequency), and $10.6 \mathrm{GHz}$ (higher frequency) are depicted in Fig. 2(a)-(c), respectively. The colors representing the current distribution in linear scale go from dark blue (weak current density) to green to yellow to red (strong current density). Fig. 2(a) shows that the electric currents are mostly concentrated on the edge portions of the top-cross-plate, the legs and around the center of the ground plane, which indicate that these elements have the most significant effect at low frequencies. Fig. 2(b) and (c) illustrates that the currents are concentrated at lower portions of the mono-cone and the ground plane center, being more important at higher frequencies, as seen in Fig. 2(c), meaning that the feed-gap (the distance between the mono-cone and the ground plane) affects the impedance matching at higher frequencies more than at middle frequencies. Note that as seen in Fig. 2 the currents on the antenna ground plane at all considered frequencies indicate that the ground size is large enough to prevent radiation from the feeding cable.

\section{EFFECTS OF Key PARAMETERS}

In this section, in order to show how the antenna was designed aiming at optimizing its performances, a parametric study was conducted using Ansoft HFSS ${ }^{\mathrm{TM}}$ electromagnetic solver. Another option would have been to use an optimizer based on for instance Genetic Algorithms [21], but here a parametric study was preferred in order to obtain better insight into the physical behavior of the antenna; while looking at the effects on the performance, only one parameter was varied at the time. The performance of the proposed antenna is affected by its geometrical parameters, i.e., top-cross-plate radiator, mono-cone, feed-gap, and ground-plane.

\section{A. Effect of the Top-Cross-Plate}

In order to understand its effect, the antenna was simulated and measured with and without (w/o) this element. Results 


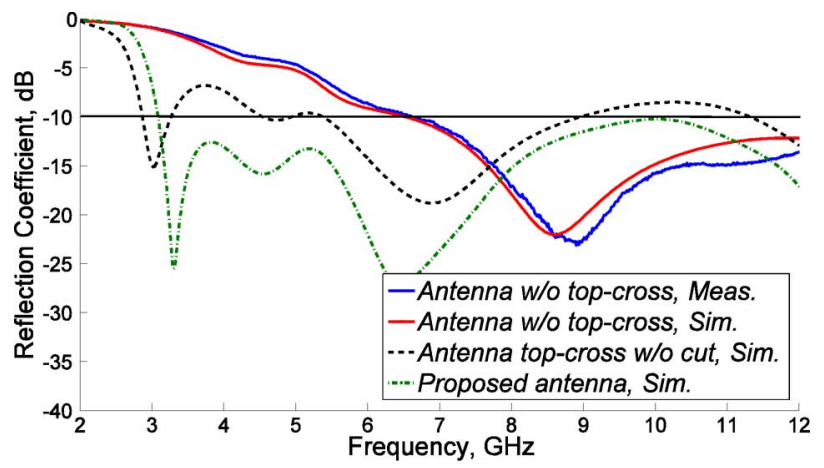

Fig. 3. Effects of the top-cross-plate on the antenna reflection coefficient.

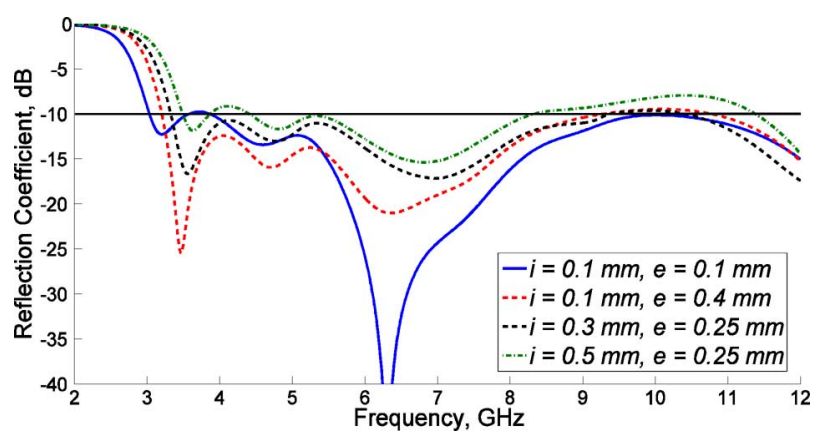

Fig. 4. Simulated antenna reflection coefficient as a function of top-cross-plate legs' dimensions.

shown in Fig. 3 confirm that the higher band $(7-12 \mathrm{GHz})$ is due to the modified mono-cone, and that the top-cross-plate produces the lower band (3-7 GHz). Moreover, to achieve wider impedance bandwidth the top-plate needs to be truncated by cutting the corners of the square-plate [" $b$ " and " $c$ " as shown in Fig. 1(a)]. It can be seen that the truncated element effectively broadens the antenna bandwidth.

\section{B. Effect of the Top-Cross-Plate Legs Dimensions (" $i$ ” “ “e”)}

By tuning the dimensions of the legs, the antenna impedance bandwidth behavior can be controlled. Parameter " $i$ " (leg thickness) was kept constant and " $e$ " (leg width) changed and then vice versa. Fig. 4 shows the antenna reflection coefficient for the variation of these two parameters. It can be seen these parameters influence mainly the lower frequency in the band.

\section{Effect of the Feed-Gap}

The feed-gap, which provides the necessary transition to the radiator, affects the antenna impedance bandwidth especially at high frequencies. Since keeping legs height fixed implies a variation of the distance between the mono-cone and the top-crossplate, the effect of the parameter " $j$ " was also investigated. As before, one parameter was fixed and the other changed. Fig. 5 shows the effects of these variations. It can be seen that small change in " $h$ ", from 0.25 to $0.1 \mathrm{~mm}$, results in a downshift from 11 to about $9 \mathrm{GHz}$ in the upper $-10 \mathrm{~dB}$ edge of frequency bandwidth. It is also seen that, without the cylinder above the mono-cone ( $j$ ' $=0$ ) the antenna impedance bandwidth decreases, while increasing " $j$ " results in an up-shift in $-10 \mathrm{~dB}$ lower frequency edge.

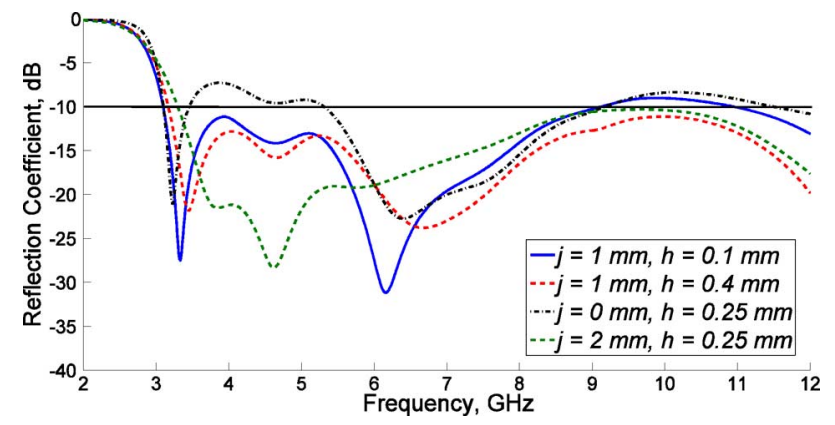

Fig. 5. Simulated antenna reflection coefficient as a function of antenna parameters "h" and "j".

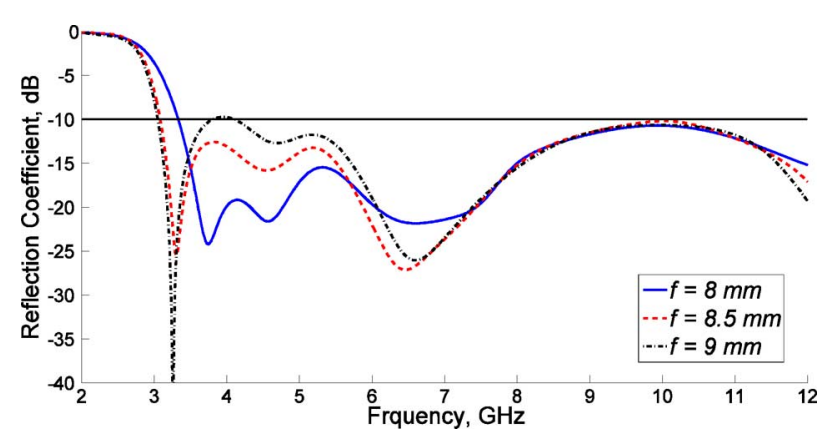

Fig. 6. Simulated antenna reflection coefficient as a function of top-cross-plate height ("f").

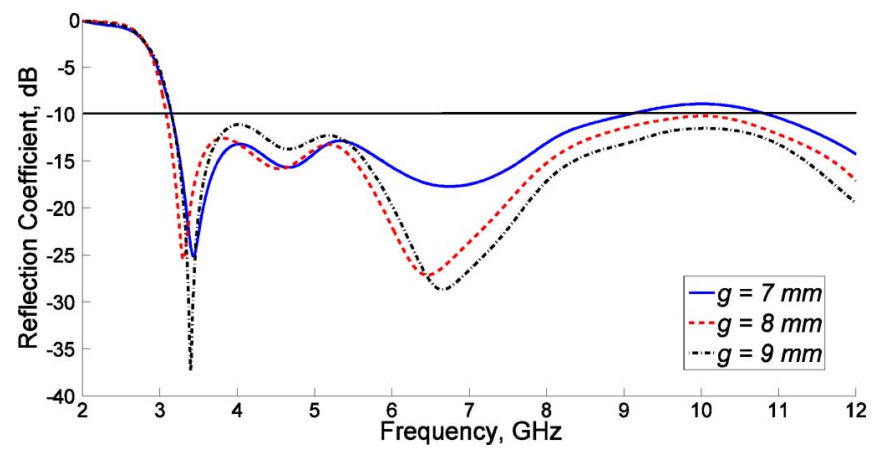

Fig. 7. Simulated antenna reflection coefficient as a function of mono-cone top radius.

\section{Effect of the Top-Cross-Plate Height}

The legs height is another important parameter that has a direct influence on the antenna impedance response. This is clearly seen in Fig. 6, where the simulated antenna reflection coefficient results for various heights are shown. It can be observed that, decreasing " $f$ " up-shifts the lower $-10 \mathrm{~dB}$ frequency edge, while increasing it causes a gradual increase of the reflection coefficient around $4 \mathrm{GHz}$.

\section{E. Effect of the Mono-Cone Top Radius}

As the top radius of the mono-cone directly influences its input impedance, the effect of this parameter on the antenna input reflection coefficient was also investigated. Simulation results depicted in Fig. 7 show that increasing or decreasing this radius leads to slightly diminishing the bandwidth. 


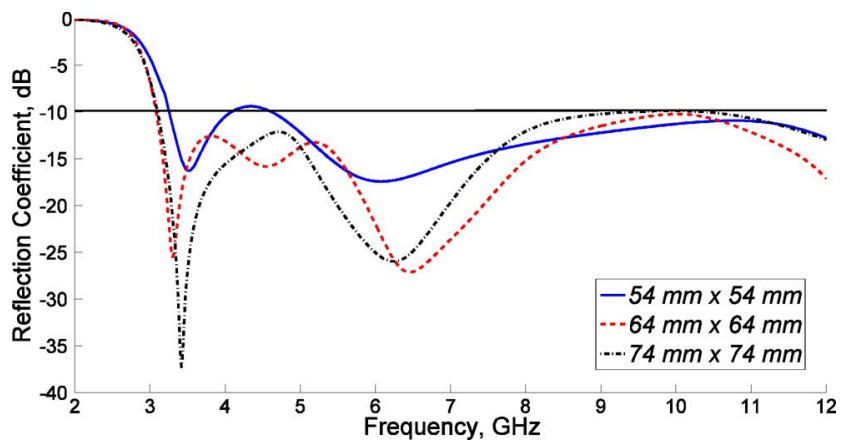

Fig. 8. Simulated antenna reflection coefficient as a function of ground-plane dimensions.

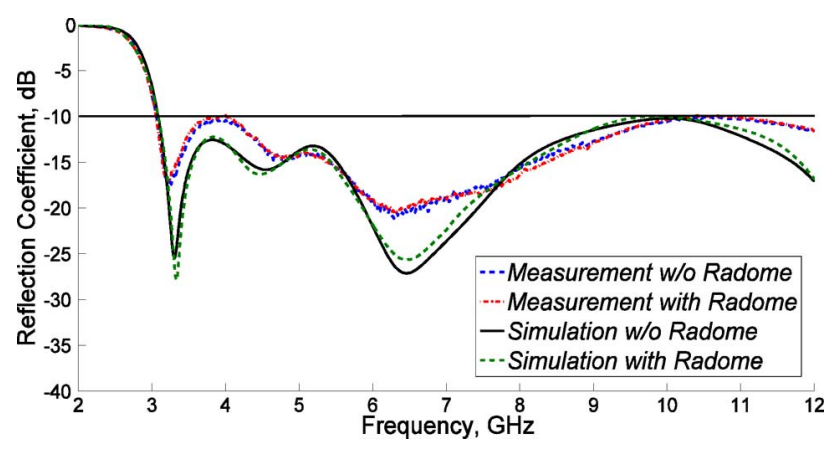

Fig. 9. Measured and simulated reflection coefficient of the proposed antenna with and without (w/o) radome.

\section{F. Effect of the Ground Plane Dimensions}

The induced surface current distributed on the antenna ground plane indicates that its dimensions affect the antenna reflection coefficient characteristic. This is seen in Fig. 8, which shows the simulated results for different ground dimensions. It is observed the ground should be at least $64 \mathrm{~mm} \times 64 \mathrm{~mm}$ to achieve the FCC defined UWB bandwidth.

\section{EXPERIMENTAL RESULTS AND DisCUSSION}

In order to validate the proposed design, both frequency- and time-domain experimental results including $S$-parameters, radiation pattern, radiation efficiency, impulse response and systemfidelity factor, are presented.

\section{A. Frequency-Domain Behavior}

The measured and simulated antenna reflection coefficients with and without the designed radome are presented in Fig. 9, showing that its $-10 \mathrm{~dB}$ bandwidth goes from 3.06 to beyond $12 \mathrm{GHz}$, and that the radome effect on matching is negligible. The measured $\left|S_{11}\right|$ confirms that the proposed antenna fully covers the ultrawide bandwidth defined by the FCC, and closely follows the simulated results.

The antenna simulated and measured radiation patterns are depicted in Fig. 10 in both $E$ - and $H$-planes at 3.1, 6.85 and $10.6 \mathrm{GHz}(x-y$ is the $H$-plane, and $y-z$ is the $E$-plane, although any vertical plane could be considered). A good agreement between measurement and simulation results is obtained. It is observed that the antenna radiates omni-directionally in the $H$-plane and fairly bi-directionally in the $E$-plane. Moreover,

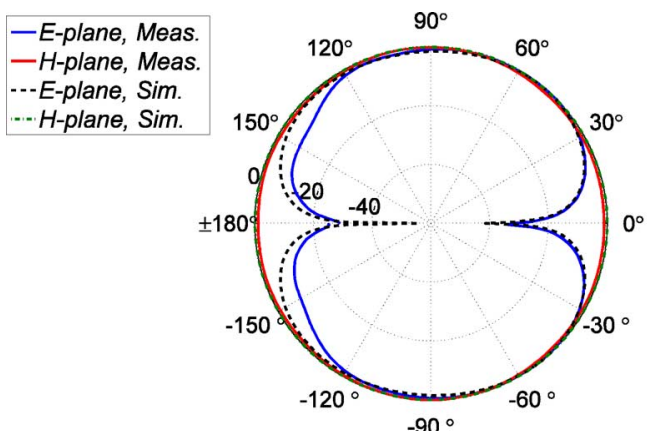

(a)

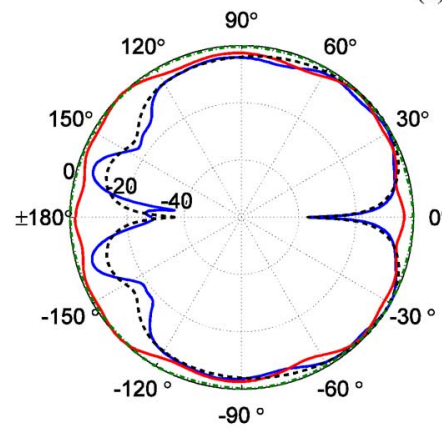

(b)

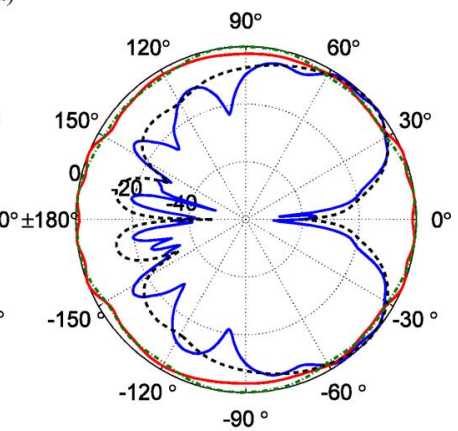

(c)
Fig. 10. Measured and simulated normalized radiation patterns of the proposed antenna at (a) $3.1 \mathrm{GHz}$, (b) $6.85 \mathrm{GHz}$, and (c) $10.6 \mathrm{GHz}$.

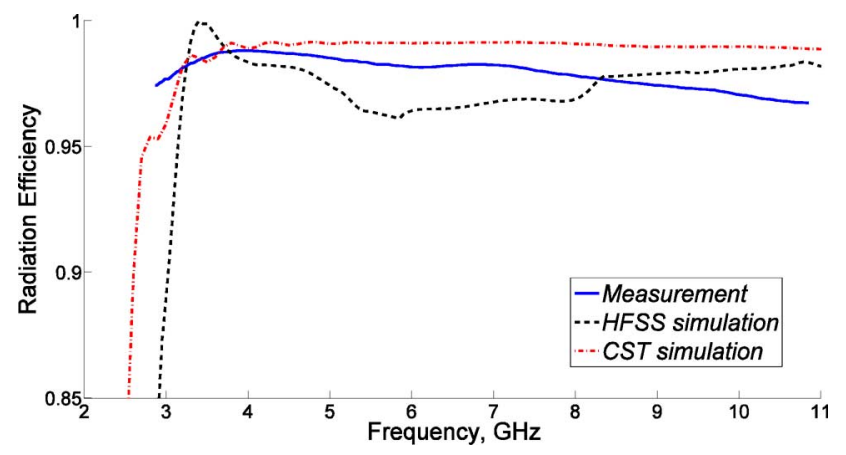

Fig. 11. Measured and simulated radiation efficiency of the proposed antenna.

these radiation properties are acceptable over the entire ultrawide bandwidth.

The antenna radiation efficiency was simulated with CST and HFSS commercial packages, and measured in a wheeler cap using the source-stirred method presented by Pires [22], [23]. The wheeler cap approach was selected owing to its accuracy for the UWB case, fast measurement, and simple postprocessing [23], [24]. The designed radome was considered in both simulations and measurements. The cylindrical cap has an internal size of $14 \mathrm{~cm} \times 20 \mathrm{~cm}$ and is built in a duralumin alloy. Fig. 11 depicts the results, showing that the proposed antenna has a radiation efficiency of $95 \%$ over its entire operating band. The agreement between the simulated and measured results is fairly good.

Since the system-fidelity factor is based on the system transfer function between two antennas placed at a separation distance, $S_{21}$ behavior needs to be considered; the setup is explained in next sub-section. The magnitude and phase of 

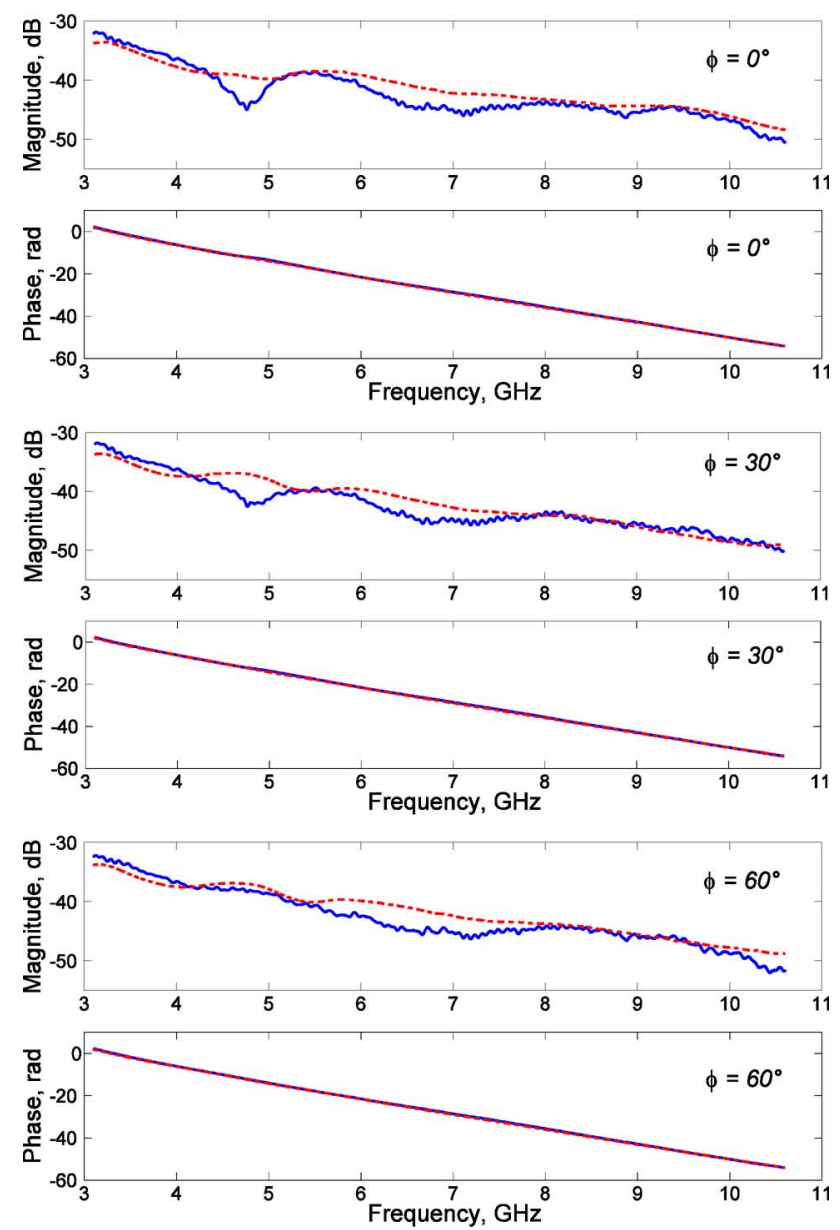

Fig. 12. $S_{21}$ magnitude and phase considering three directions in the azimuthal plane: measured (solid-blue), simulated (dash-red).

the measured and HFSS simulated $S_{21}$ are shown in Fig. 12, considering three different directions in the azimuthal plane. It is observed that $S_{21}$ magnitude varies smoothly and its phase is almost linear over the frequency range of interest. The latter is desirable in order to obtain almost constant group delay which is vital for the transmission and reception of nanoseconds pulses. Note that the simulated and measured results are in a good agreement.

\section{B. Time-Domain Behavior}

The time-domain setup selected in this paper comprises two identical antennas, one for transmitting (Tx) and one for receiving $(\mathrm{Rx})$, placed side-by-side with a large enough separation distance $(30 \mathrm{~cm})$ to be considered in the far-field of each other. Measurements were performed in an anechoic chamber to eliminate multipath reflections from surrounding scatterers. To investigate the system behavior in different directions, the two antennas time performance was measured in different orientations $\left(\varphi=0^{\circ}, 30^{\circ}\right.$, and $\left.60^{\circ}\right)$; the Tx antenna is fixed while the $\mathrm{Rx}$ antenna rotates in the azimuthal plane.

The time behavior of a UWB antenna can be obtained either from time- or frequency-domain measurements. In the time-domain, using ultra short pulses, measurements are faster than in the frequency-domain making use of the Fourier transform. However, frequency-domain measurements have the advantage

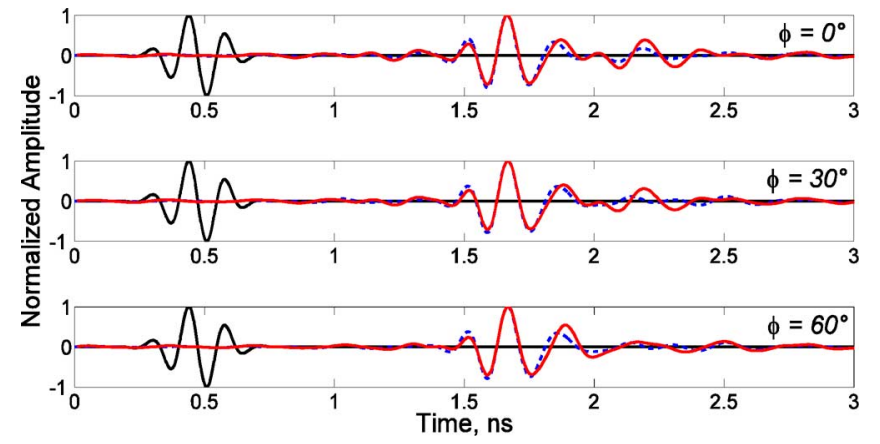

Fig. 13. Tx and Rx signals: input (solid-black), simulated (dash-blue), measured (solid-red).

TABLE II

Simulated AND MEASURED SYSTEM FIDELITy FACtoR

\begin{tabular}{c|c|c}
\multirow{2}{*}{$\varphi$} & \multicolumn{2}{|c}{ System-Fidelity Factor } \\
\cline { 2 - 3 } & Simulated & Measured \\
\hline $0^{\circ}$ & 0.862 & 0.741 \\
\hline $30^{\circ}$ & 0.828 & 0.751 \\
\hline $60^{\circ}$ & 0.829 & 0.708
\end{tabular}

of high dynamic range and, moreover, there is the ability to describe the radiation of arbitrary waveforms [25]. Thus, in order to have the transient behavior, time-domain data were extracted from frequency-domain measurements using the inverse fast Fourier transform (IFFT). In this study, the simulated impulse responses were also extracted from HFSS frequency-domain $S_{21}$ data.

Due to the mentioned advantage of describing the radiation of arbitrary input signals, here, the CST default Gaussian modulated pulse with spectrum corresponding to $3.1-10.6 \mathrm{GHz}$ was used as the input signal, as it completely complies with the FCC indoor and outdoor power masks [26].

To assess the quality of the received pulse and to be able to compare directly with measurements, the system-fidelity factor [25], which reflects the correlation between the input and the received pulse, was calculated rather than the fidelity factor. It is worth mentioning that the system-fidelity factor takes into consideration the distortion induced by the two antennas (Tx and $\mathrm{Rx})$, whereas the fidelity factor takes into account the transmit antenna effect only [25]. The system fidelity factor is thus always lower than the fidelity factor, but has the advantage to be a measureable quantity. Fig. 13 shows the antenna impulse response in the three considered directions. The perfect overlap of the simulated and measured Rx signals shows good prediction of the transient performance of the antenna for the chosen orientations. To quantify the level of distortion, the calculated system-fidelity factor results are given in Table II. The agreement between the simulated and measured values is reasonable; the differences are mainly due to the $S_{21}$ magnitude variation. As it is shown in Fig. 12, $S_{21}$ is linear in phase and has less magnitude variation in simulation than in measurement, thus, higher system-fidelity values in simulation as compared to measurement are expected. This is confirmed by results shown in Table II. Nevertheless, the system-fidelity factor results indicate that the level of signal distortion is quite acceptable $(>0.5)$ for the transmission of UWB signals. The designed radome has negligible effects on the time-domain performance. 


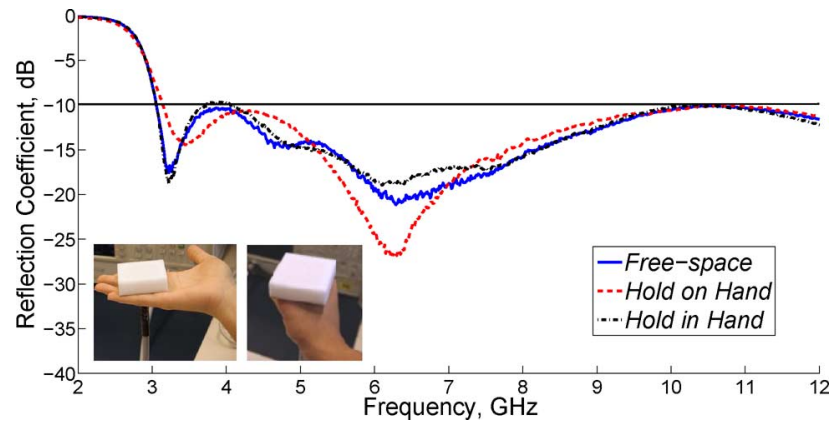

Fig. 14. Measured antenna reflection coefficient when holding it on an openhand and in a hand grabbing the feeding cable.

\section{BODY EFFECTS}

The applications of UWB-WBAN antennas include human proximity operation. A preliminary study of the influence of such scenarios on the antenna was conducted. The reflection coefficient of the proposed antenna was measured in two human proximity situations as shown in Fig. 14, holding it on an openhand and grabbing the feeding cable. The tests show that, in spite of maximum expected influence of the body, the antenna does not significantly detune in both scenarios. This was expected as the antenna is vertically polarized, and was designed to have a large enough ground plane to avoid radiation from the feeding cable. Note that although proximity effects are expected to be low in UWB antennas, due to their low-Q nature [27], [28], they can be sensitive to the proximity of the human body [9], [29], [30]. It should be mentioned that for realistic body proximity scenarios the antenna time behavior is expected to improve [10], [11]. Thus, the results obtained in the considered scenarios clearly show the suitability of this highly efficient low-profile antenna for WBAN applications.

\section{CONCLUSION}

A novel low-profile vertically-polarized UWB antenna is presented. The antenna consists of a modified mono-cone with a top-cross-plate overhead, having a small overall height of $8.5 \mathrm{~mm}$. The salient antenna parameters were investigated aiming at an optimal design to obtain the best UWB performance and the smallest height. The parametric studies also showed that this antenna is not very sensitive to manufacturing tolerances. Measured results confirm that the proposed antenna, including the designed radome, provides an ultrawide bandwidth (3.06 to $12 \mathrm{GHz}$ ), has omni-directional radiation properties in the $H$-plane validated over the entire FCC UWB frequency band, has a radiation efficiency over $95 \%$, and has an acceptable system-fidelity factor for the transmission of the UWB signals. The radome effects on the antenna performance were found to be negligible. Furthermore, the antenna does not detune in presence of the body, which is mainly due to its polarization and the adequate ground size. These results indicate that the proposed antenna is a suitable candidate for future WBAN applications.

\section{ACKNOWLEDGMENT}

The authors would like to thank A. Almeida, C. Brito, and N. Pires for their help with the measurements.

\section{REFERENCES}

[1] P. S. Hall and Y. Hao, Antennas and Propagation for Body Centric Communications Systems. Norwood, MA, USA: Artech House, 2006.

[2] P. S. Hall and Y. Hao, "Antennas and propagation for body centric communications," presented at the 1st EuCAP, Nice, France, 2006.

[3] E. M. Staderini, "UWB radars in medicine," IEEE Aerosp. Electron. Syst. Mag., vol. 17, no. 1, pp. 13-18, Jan. 2002.

[4] W.-T. Chen and H.-R. Chuang, "Numerical computation of human interaction with arbitrarily oriented superquadric loop antennas in personal communications," IEEE Trans. Antennas Propag., vol. 46, no. 6, pp. 821-828, Jun. 1998 .

[5] A. Alomainy, A. Sani, A. Rahman, J. G. Santas, and Y. Hao, "Transient characteristics of wearable antennas and radio propagation channels for ultrawideband body-centric wireless communications," IEEE Trans. Antennas Propag., vol. 57, no. 4, pp. 875-884, Apr. 2009.

[6] N. Chahat, M. Zhadobov, R. Sauleau, and K. Ito, "A compact UWB antenna for on-body applications," IEEE Trans. Antennas Propag., vol. 59, no. 4, pp. 1123-1131, Apr. 2011.

[7] M. Klemm, I. Z. Kovcs, G. F. Pedersen, and G. Troster, "Novel small size directional antenna for UWB WBAN/WPAN applications," IEEE Trans. Antennas Propag., vol. 53, no. 12, pp. 3884-3896, Dec. 2005.

[8] A. M. Abbosh, "Miniaturization of planar Ultrawideband antenna via corrugation," IEEE Antennas Wirel. Propag. Lett., vol. 7, pp. 685-688, 2008.

[9] M. Koohestani, N. Pires, A. K. Skrivervik, and A. A. Moreira, "Bandwidth enhancement of a wearable UWB antenna near a human arm," Microw. Opt. Technol. Lett., vol. 55, no. 12, pp. 2965-2967, 2013.

[10] T. S. P. See and Z.-N. Chen, "Experimental characterization of UWB antennas for on-body communications," IEEE Trans. Antennas Propag., vol. 57, no. 4, pp. 866-874, Apr. 2009.

[11] M. Koohestani, N. Pires, A. K. Skrivervik, and A. A. Moreira, "Performance study of a UWB antenna in proximity to a human arm," IEEE Antennas Wirel. Propag. Lett., vol. 12, pp. 555-558, 2013.

[12] H. B. Lim, D. Baumann, J. Cai, R. Koh, E. P. Li, and Y. Lu, "Antennae polarization for effective transmission of UWB signal around human body," in Proc. IEEE Int. Conf. Ultra-Wideband, 2007, pp. 220-224.

[13] C.-H. Kang, S.-J. Wu, and J.-H. Tarng, "A novel folded UWB antenna for wireless body area network," IEEE Trans. Antennas Propag., vol. 60, no. 2, pp. 1139-1142, Feb. 2012.

[14] D.-D. Cara, R. Torres, J. Trajkovikj, J.-F. Zürcher, and A. K. Skrivervik, "A low-profile UWB antenna for wearable applications: The Tripod kettle antenna (TKA)," presented at the 7th EuCAP, Gothenburg, Sweden, 2013.

[15] B. Sanz-Izquierdo, J. C. Batchelor, and M. Sobhy, "UWB wearable button antenna," presented at the 1st EuCAP, Nice, France, 2006.

[16] H. S. Chan, H. I. Hraga, R. A. Abd-Alhameed, N. J. McEwan, J. M. Noras, and P. S. Excell, "A low-Profile Ultra-Wideband modified planar inverted-F antenna," IEEE Trans. Antennas Propag., vol. 61, no. 1, pp. 100-108, 2013.

[17] Y. Song-Tao, L. Ying, W. Jiang, and S.-X. Gong, "A novel textile UWB antenna," in Proc. Int. Symp. Antennas Propag., 2012, pp. 1051-1054.

[18] S.-T. Yu, Y. Liu, W. Jiang, S.-X. Gong, and T. Hong, "A novel compact UWB textile button antenna," Progr. Electromagn. Res. C, vol. 32, pp. 233-243, 2012.

[19] R. Bourtoutian, C. Delaveaud, and S. Toutain, "Low profile UWB shorted dipole antenna," in Proc. IEEE Int. Symp. Antennas and Propag. Soc., 2007, pp. 5729-5732.

[20] W. Feng, K. Z. -fan, and L. D. -hai, "An novel design for an ultra-wide band antenna," in Proc. Int. Symp. Antennas, Propag. \& EM Theory, 2006, pp. 1-5.

[21] Y. Rahmat-Samii and E. Michielssen, Electromagnetic Optimization by Genetic Algorithms. Hoboken, NJ, USA: Wiley, 1999.

[22] N. Pires, C. Mendes, M. Koohestani, A. K. Skrivervik, and A. A. Moreira, "Novel approach to the measurement of Ultra-wideband efficiency," IEEE Antennas Wirel. Propag. Lett., vol. 12, pp. 1512-1515, 2013.

[23] N. Pires, C. Mendes, M. Koohestani, A. K. Skrivervik, and A. A. Moreira, "Radiation efficiency of a coplanar-fed ultra-wideband antenna," in Proc. IEEE Int. Symp. Antennas and Propag. Soc., 2012, pp. 1-2.

[24] H. Schantz, The Art and Science of UWB Antenna. Norwood, MA: Artech House, 2005.

[25] G. Quintero, J.-F. Zürcher, and A. K. Skrivervik, "System fidelity factor: A new method for comparing UWB antennas," IEEE Trans. Antennas Propag., vol. 59, no. 7, pp. 2502-2512, Jul. 2011.

[26] M. Koohestani, N. Pires, A. K. Skrivervik, and A. A. Moreira, "Timedomain performance of patch-loaded band-reject UWB antenna," IEE Electronics Lett., vol. 49, no. 6, pp. 385-386, 2013. 
[27] T. Yang, W. A. Davis, W. L. Stutzman, and W. C. Huynh, "Cellularphone and hearing-aid interaction: An antenna solution," IEEE Antennas Propag. Mag., vol. 50, no. 3, pp. 51-65, Mar. 2008.

[28] T. Yang, W. A. Davis, and W. L. Stutzman, "Fundamental-limit perspectives on ultrawideband antennas," Radio Sci., vol. 44, pp. 1-8, 2009.

[29] T. S. P. See, Z. N. Chen, and X. M. Qing, "Proximity effect of UWB antenna on human body," in Proc. Microwave Conf., 2009, pp. 2192-2195.

[30] T. Tuovinen, T. Kumpuniemi, K. Y. Yazdandoost, M. Hamalainen, and J. Iinatti, "Effect of the antenna-human body distance on the antenna matching in UWB WBAN applications," in Proc. Int. Symp. Medical Inf. Commun. Technol., 2013, pp. 193-197.

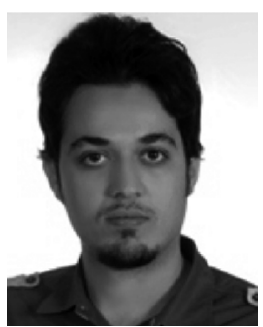

Mohsen Koohestani received the B.Sc. and M.Sc. degrees in electrical engineering from the Islamic Azad University, Tehran, Iran, in 2007 and 2010, respectively. $\mathrm{He}$ is currently pursuing the $\mathrm{PhD}$ degree jointly at the Ecole Polytechnique Fédérale de Lausanne, Lausanne, Switzerland, and the Universidade de Lisboa, Lisbon, Portugal.

His main field of research includes novel design and analysis of UWB antennas for WBAN applications, antenna interactions with human body, computational electromagnetics (time- and frequency-domain methods), and microwave circuits and components. He is the author or co-author of about 30 peer-reviewed scientific journal and conference papers.

Mr. Koohestani was awarded a study abroad scholarship in 2011 to continue his Ph.D. studies.

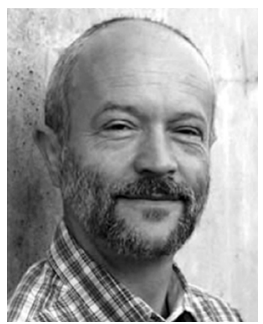

J.-F. Zürcher was born in Vevey, Switzerland, in 1951. He graduated with the degree of Electrical Engineer from the Ecole Polytechnique Fédérale de Lausanne (EPFL), Lausanne, Switzerland, in 1974.

$\mathrm{He}$ is presently employed as a permanent Scientific Associate with the Laboratoire d'Electromagnétisme et d'Acoustique EPFL, where he is the Manager of the Microwave Laboratory. His main interest lies in the domain of microstrip circuits and antennas. In 1988, he invented the SSFIP concept ("Strip Slot Foam Inverted Patch antenna"), which became a commercial product. He is presently developing instrumentation and techniques for the measurement of near fields of planar structures and microwave materials measurement and imaging. He is the author or coauthor of about 180 publications, chapters in books and papers presented at international conferences. He is a coauthor of the book Broadband Patch Antennas (Artech House, 1995). He holds nine patents.

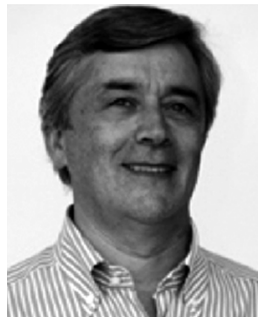

Antonio A. Moreira was born in Aveiro, Portugal, in 1952 . He received the Ph.D. degree in electrical and computer engineering from the Instituto de Telecomunicações, Instituto Superior Técnico, Universidade Técnica de Lisboa, Lisbon, Portugal, in 1984 where he is currently an Associate Professor in the subjects of antennas and radar.

$\mathrm{He}$ is a member of Instituto de Telecomunicações where he performs his research activity in the IT Wireless group with his present interest focused in antennas for mobile communications. He has participated in the RACE Projects "Multigigabit Transmission in the Subscriber Loop" and "MODAL - Microwave Optical Transmission Antenna Link", and in the IST projects "ACE - Antenna Center of Excellence", "FLOWS Flexible Convergence of Wireless Standards and Services", and "NEWCOM++ - Network of Excellence in Wireless Communications ++", - EU-FP7 ICT 20072010. He is an active member of EurAAP Working Group on Small Antennas (WG7). He is the author or coauthor of about 70 publications, chapters in books, and papers presented at international conferences.

Dr. Moreira was the General Chair of "IEEE iWAT2010 - International Workshop on Antenna Technology", held in Lisbon, Portugal, in March 2010.

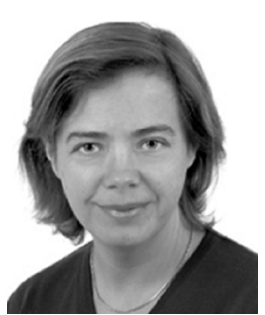

Anja K. Skrivervik received the Electrical Engineering degree and $\mathrm{Ph} . \mathrm{D}$. degree from Ecole Polytechnique Fédérale de Lausanne (EPFL), Lausanne, Switzerland, in 1986 and 1992, respectively.

After a stay at the University of Rennes, Rennes, France, as an Invited Research Fellow and two years in the industry, she returned part time to EPFL as an Assistant Professor in 1996, where she is now a Professeur Titulaire. Her teaching activities include courses on microwaves and antennas. Her research activities include electrically small antennas, implantable and on body antennas, multifrequency and ultrawideband antennas, numerical techniques for electromagnetics, and microwave and millimeter wave MEMS. She is the author or coauthor of more than 100 scientific publications. She is very active in European collaboration and European projects.

Dr. Skrivervik was the Chairperson of the Swiss URSI from 2003 to 2011. 